\title{
Design of an Optimal High Pass Filter in Frequency - Wave Number $(F-K)$ Space for Suppressing Dispersive Ground Roll Noise from Onshore Seismic Data
}

\author{
Adizua, O. F. , Inchinbia, S., Ekine, A. S. \\ Department of Physics/Applied Geophysics, Faculty of Science, University of Port Harcourt, Nigeria
}

Copyright $(2017$ by authors, all rights reserved. Authors agree that this article remains permanently open access under the terms of the Creative Commons Attribution License 4.0 International License

\begin{abstract}
The ultimate goal in seismic signal processing (pre- processing or main processing) is to enhance the signal to noise ratio (S-N-R). In this study, two high pass (low cut frequency) filters were designed in frequency - wave number $(F-K)$ space and applied to onshore 3D seismic data acquired from a typical Niger Delta field to suppress dispersive ground roll in a bid to enhance S-N-R. The seismic shot records were initially displayed to show the seismic data ensembles in the conventional offset - time $(X-T)$ domain. A frequency - wave number spectrum was then created using a VISTA software algorithm to analyze the spectral patterns of the signals and noise. Two high pass filters were then designed in the $F-K$ space to mute the low frequency ground roll from the data. The filters were then applied to the entire seismic data ensemble for the filtering operation. The results obtained after the two filtering operations were compared to ascertain the optimal filter which was most effective for the suppression of the unwanted ground roll noise.
\end{abstract}

Keywords $F-K$ Space, $F-K$ Spectral Analysis, Dispersive Ground Roll, High Pass Filter, Muting

\section{Introduction}

One of the major objectives of seismic data processing is to effectively identify and suppress seismic energies that do not correspond to primary body waves. These include mainly energy from surface waves (ground rolls), which do not travel deep into the earth and hence do not carry any useful information about the deeper subsurface. Most times, the signals recorded at the end of a seismic acquisition is a combination of energies from body waves which have traversed through the shallow and deep subsurface together with some of these unwanted energies which we generally term as noise. This noise interferes with and masks the deep subsurface signal in seismic shot gathers [1].

Ground rolls are characterized by low frequencies, low velocity and their high amplitudes on seismic records. They travel along the surface of the earth with velocity/frequency dispersion. Their dispersive nature makes them to mask the shallow reflections, at short offsets, and deep reflections, at long offsets [2, 3]. Ground rolls most often constitute a problem for onshore seismic datasets since they do not carry useful information about the deep surface which most times is the target of interest. Suppressing them, therefore, from seismic datasets is critical for enhanced subsurface imaging. Different methods are applied in seismic data processing to suppress ground roll noise while attempting to preserve the primary reflected signals and their amplitudes. These methods are based on the possibility of separating the signal and noise based on their distinguishing characteristics such as frequency content or apparent move out [4].

In separation of signal (primary body waves) from noise (ground rolls), it has been recognized that the data can be transformed into alternative domains, such as the Tau- $P, F-K$ and $F-X$ domains from the conventional offset - time $(T-X)$ domain, where the noise is separated from the signal groups and can thus be suppressed without necessarily corrupting the integrity of the primary signals. The data is then inverse-transformed back to the original domain $(T-X)$ for further processing. It is important to note that an ideal transform should successfully suppress the noise component with no or very minimal distortion of the signal and should leave no footprint behind. A number of research works on ground roll noise suppression in onshore seismic data have been carried out and results carefully documented. R. N. Pertiwi and S. Winardhie [7] proposed a method of a redesigned Optimum Wiener filtering method which they used to extract ground-roll noise. For estimating the ground-roll in seismic dataset they worked on, they created a reference noise trace as the initial model of ground-roll using a sweep function. The success level of their method depended on the parameters that are used in building the reference noise trace, especially frequency interval. For this reason, they considered the amplitude spectra of extracted ground-roll noise that are derived from various frequency 
intervals to know the most appropriate frequency interval to select. R. Kappius and G. Crews [8] suppressed ground roll noise in three component seismic data using adaptive filters. Adaptive filtering enabled them to apply single receiver station filters using correlation between components to identify and remove noise with non-stationary filters. The polarized, band limited nature of ground rolls allowed them to differentiate it from other signals in the station components to aid in designing optimal filters. From the results they obtained there was a significant ground roll reduction without any dependence on spatial sampling or receiver arrays.

Our primary motivation for this study is to demonstrate the effectiveness of using the conventional Frequency - Wave number $(F-K)$ space or domain filtering technique in suppressing ground roll noise in an attempt to improve the signal to noise ratio (SNR) of 3D onshore seismic datasets acquired from a field lying within the south western part of the Niger Delta Basin, Nigeria. Two different high pass filters were conceptualized and designed in the $F-K$ space and applied to the same seismic dataset in a bid to ascertain the optimal filter which achieves the best ground roll noise suppression. The strategy adopted for the suppression of the ground roll noise from the seismic datasets was to design two high pass filters in the frequency - wave number $(F-K)$ space which mutes off low frequencies and applying it to the datasets. After the filtering operation, the filtered output were then transformed back to the conventional offset - time domain to an appreciable extend ground roll noise free. Both noise suppression outputs were then compared to ascertain the optimal filter that achieved the best noise suppression result.

\subsection{Location and Outline Geology of the Study Area}

The study area is located on the onshore part of SouthSouth Niger Delta, South-East of Nigeria. The field lies in Ohaji - Egbema LGA, a few kilometers from Owerri in Imo State (Figure 1). The Niger delta is underlain mainly by three stratigraphic units, the deepest Akata Formation, the middle Agbada Formation and the top Benin Formation. The Benin Formation is made up mainly of continental sand deposits with intercalation of shale and is covered with topmost low velocity layer which, in most cases, is weathered within which surface waves are excited and generated. Immediately below the Benin Formation is the reservoir sand of the Agbada Formation which is believed to house the oil and gas resource of the Niger Delta. The Akata Formation is also believed to be the source rock for the petroleum resource and also hydrocarbons reservoirs in deep offshore.

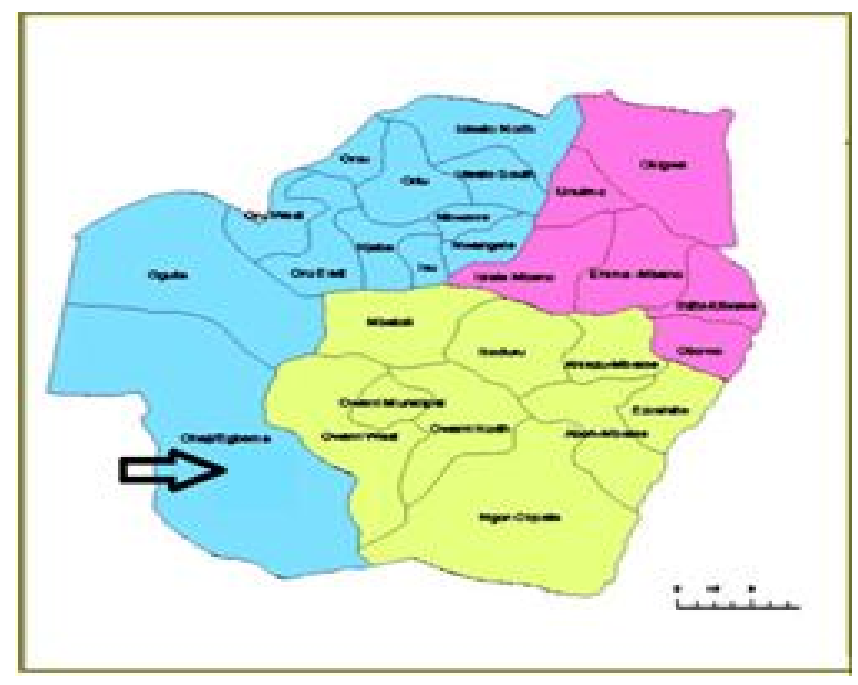

Figure 1. Map of Imo State showing the Location of Study Area

\section{Data Presentation, Methodology and Processing Workflow}

The seismic shot record used for the study (Figure 2) is an unprocessed 3D seismic data acquired from an onshore field in the southern part of the Niger delta basin and is presented in the conventional Offset - Time domain $(X-T)$. The data is an ensemble of signals recorded from shot 102500 by different receivers presented in common shot configuration. The full shot spread is continuous and so long and could not have possibly been displayed in its full length, which is the reason only a panel from the full signal spread or ensemble was selected for the study. This shot was selected from the full data spread with the aid of a Vista flow command and our choice of shot 102500 was due to the fact that it contained significant amount of ground roll noise imprints which we intend to suppress. 


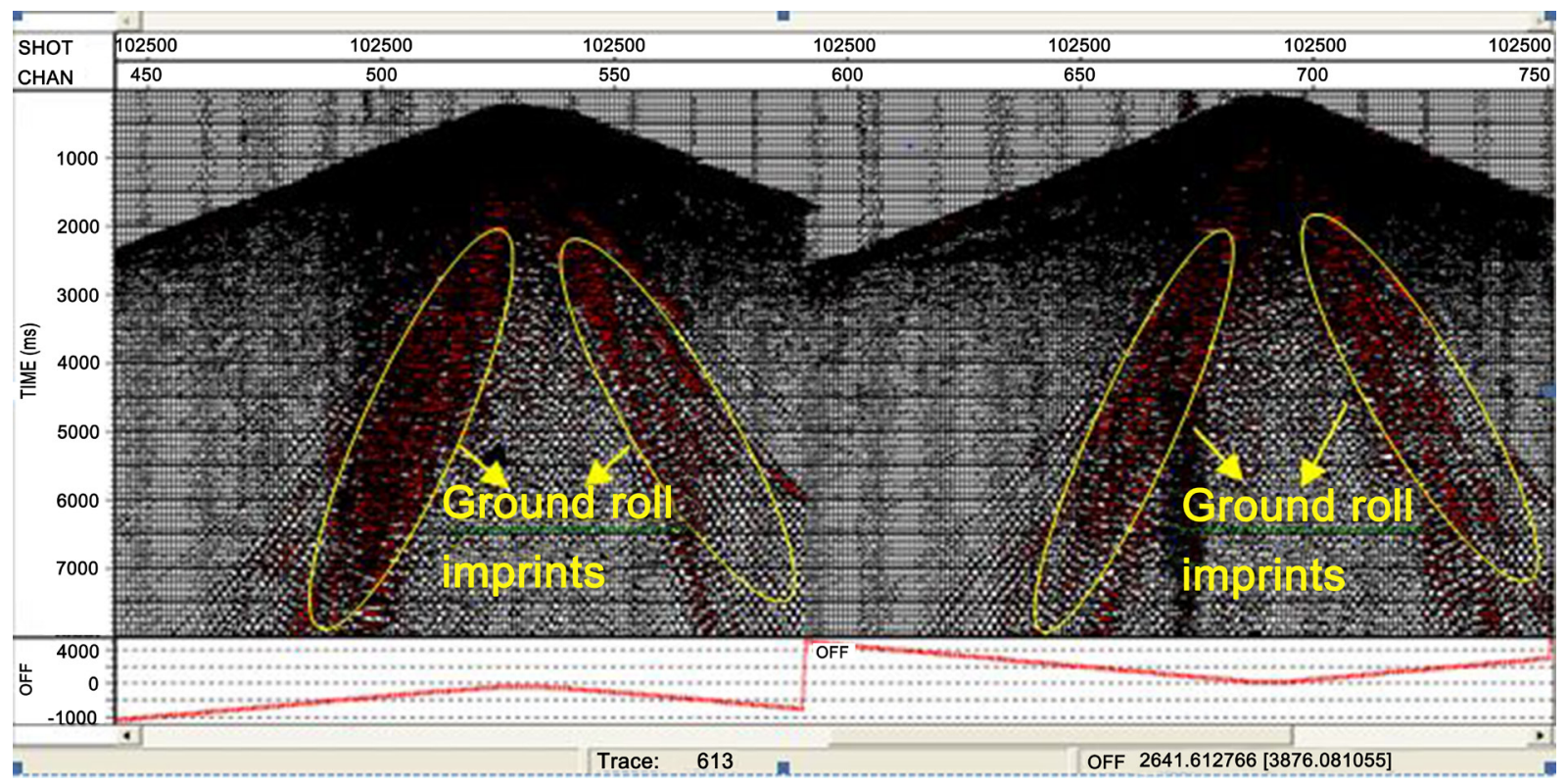

Figure 2. Input unprocessed data showing ground roll noise imprints

The Ground roll noise suppression was carried out by high pass filters designed in the frequency- wave number $(F-K)$ space. The underlying principle of filtering in this space or domain is briefly explained below.

\subsection{The Principle of Filtering in 2D Frequency - Wave Number $(F-K)$ Space}

The transformation of seismic data from the $t-x$ domain to the $f-k$ domain is achieved by the 2-D Fourier transform. The 2-D Fourier transform is able to separate events according to their dips in the $f-k$ domain [5]. A linear event, $f(t, x)$, can be described by equation (1).

$$
\mathrm{f}(\mathrm{t}, \mathrm{x})=\mathrm{s}(\mathrm{t}) * \delta(\mathrm{t}-\tan (\alpha) \mathrm{x}+\mathrm{b})
$$

where the symbol "**" denotes convolution with respect to the variable time $(\mathrm{t}), \mathrm{s}(\mathrm{t})$ is seismic wavelet, $\alpha$ is the angle between the simulated linear event and the space axis, and the constant $b$ is the intercept of the event on the time axis. The 2-D Fourier transformed expression of equation (1) is given as

$$
\mathrm{F}(\omega, \mathrm{k})=\mathrm{S}(\omega) \mathrm{e}^{\mathrm{i} \omega \mathrm{b}} \delta(\mathrm{k}-\omega \tan (\alpha))
$$

where $S(\omega)$ is the Fourier transform of the time function $s(t)$ in equation (1). Equation (2) shows that any linear event in the $t-x$ domain can be transformed into another linear event in the $f-k$ domain [5]. Linear events such as ground roll noise in the $\mathrm{t}-\mathrm{x}$ domain can be separated in the $f-k$ domain by dip [6]. Based on this property of the 2-D Fourier transform, two high pass (low cut frequency) filters were designed in the $f-k$ space or domain to suppress the unwanted ground roll noise imprints from the seismic dataset. The designed filter were then applied to the data in the $f-k$ space to mute the noise and eventually the data was transformed back to its original offset - time domain now to a very large extent noise free. The results of the two filtering operations were compared to ascertain the optimal filter in terms of the best noise suppression result. In processing, the workflow adopted was a display of the seismic shot record in the offset - time $(X-T)$ domain showing the noise imprints, followed by the transformation of the data to the frequency - wave number $(F-K)$ space from where a spectral analysis window was created to analyze the data. Two high pass (low cut frequency) filters were then designed in this $F-K$ spectral analysis window and applied to the data after which a re-transformation of the data from the $F-K$ domain back to the $X-T$ domain was achieved by carrying out an inverse 2D Fourier transform operation on the datasets. 


\section{Results Presentation}

\subsection{First Procedure/ $1^{\text {st }} F-K$ Filter Design}

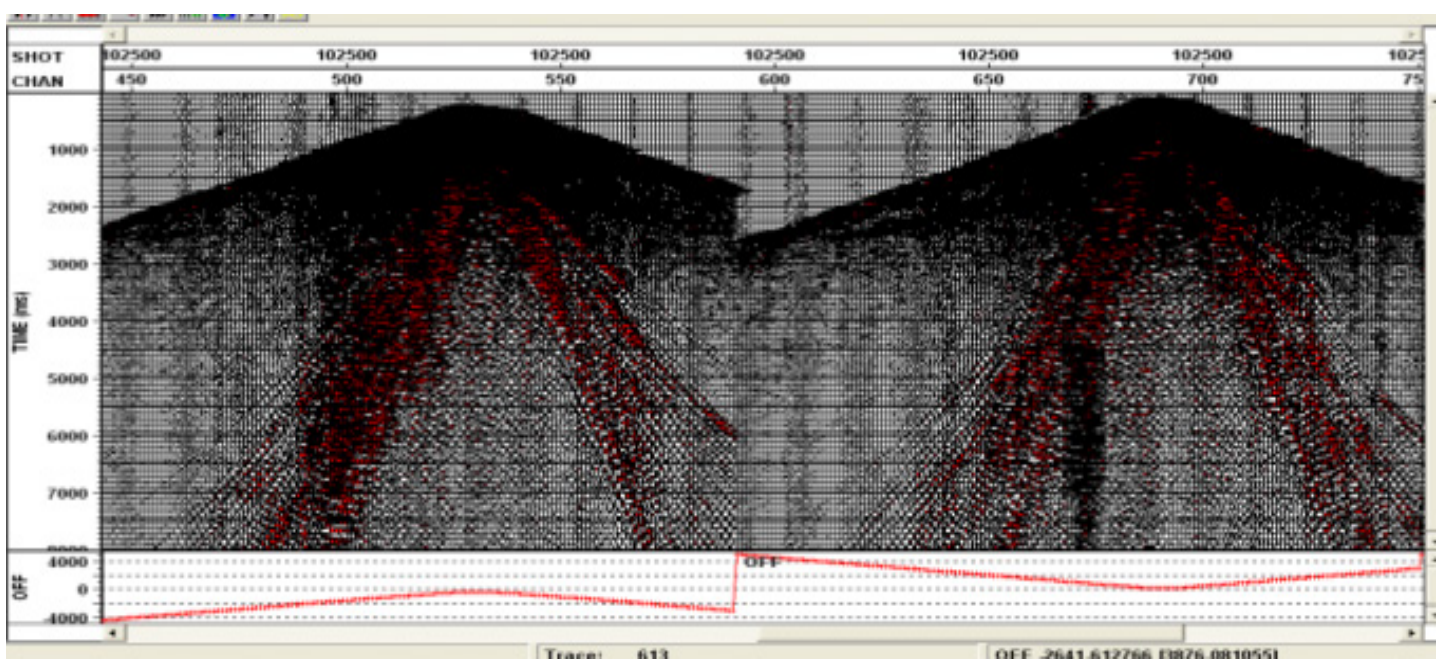

(a)

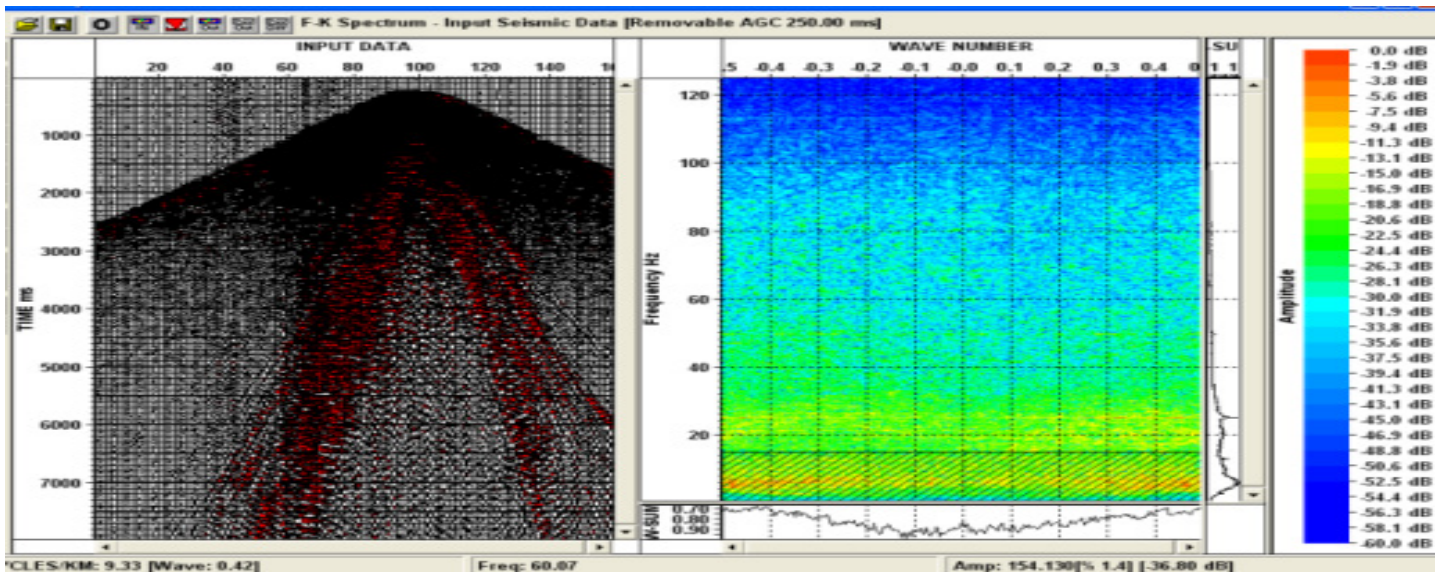

(b)

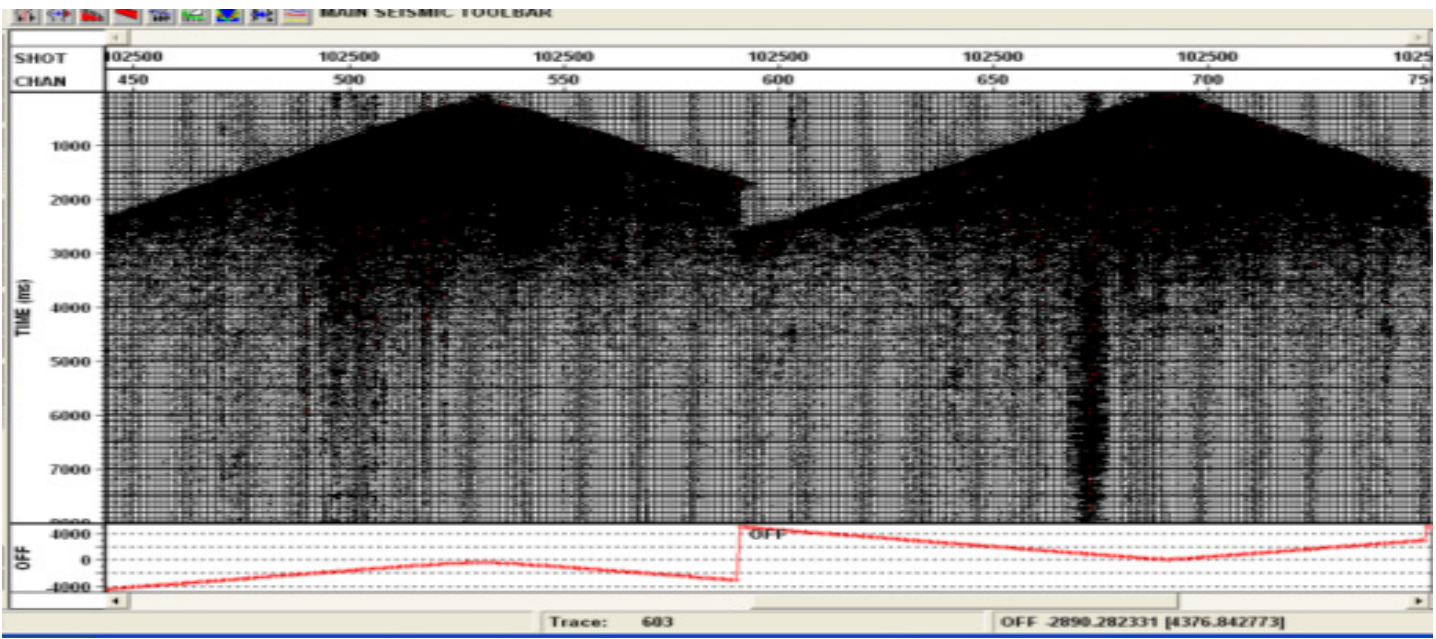

(c)

Figure 3. (a) Shows the input data with ground roll noise imprints. (b) Shows the $1^{\text {st }}$ high pass filter designed in the $F-K$ space juxtaposed with a panel of input data. (c) Shows the noise suppression result after the filtering operation. 


\subsection{Second Procedure/ $2^{\text {nd }} F-K$ Filter Design}

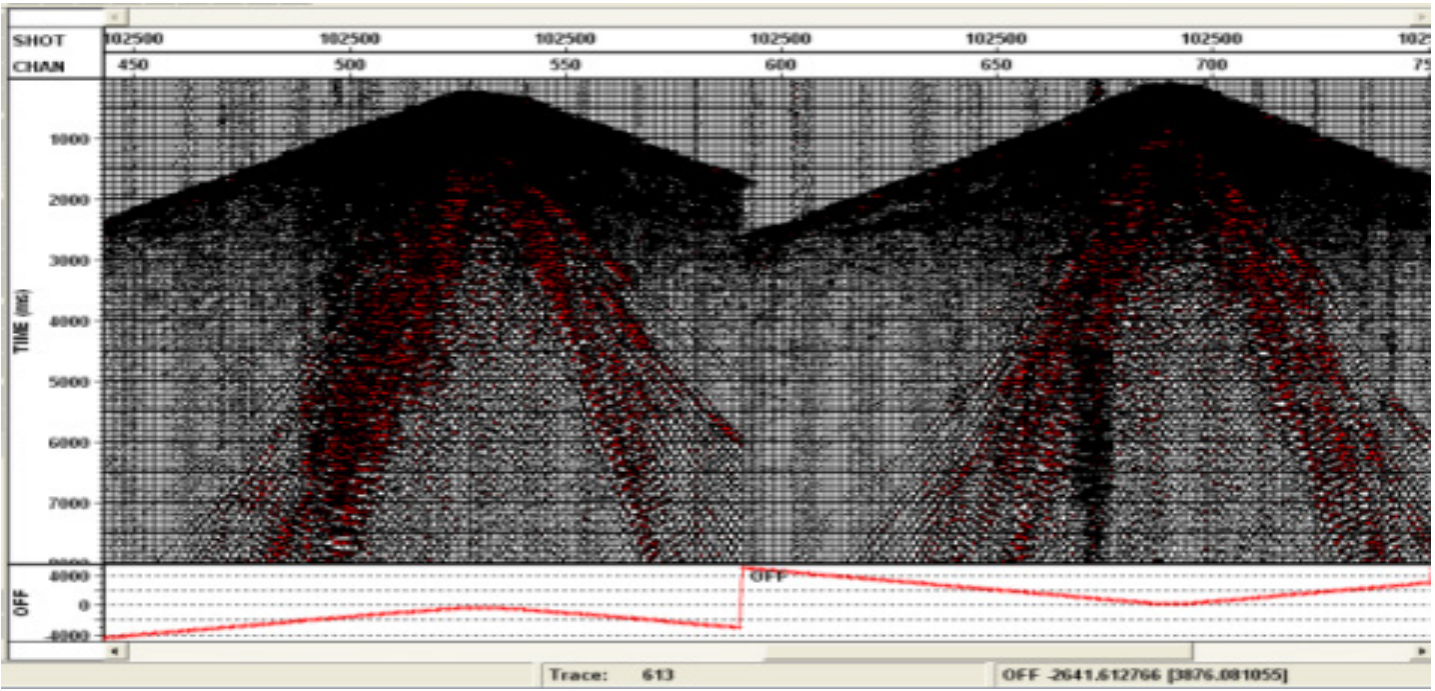

(a)

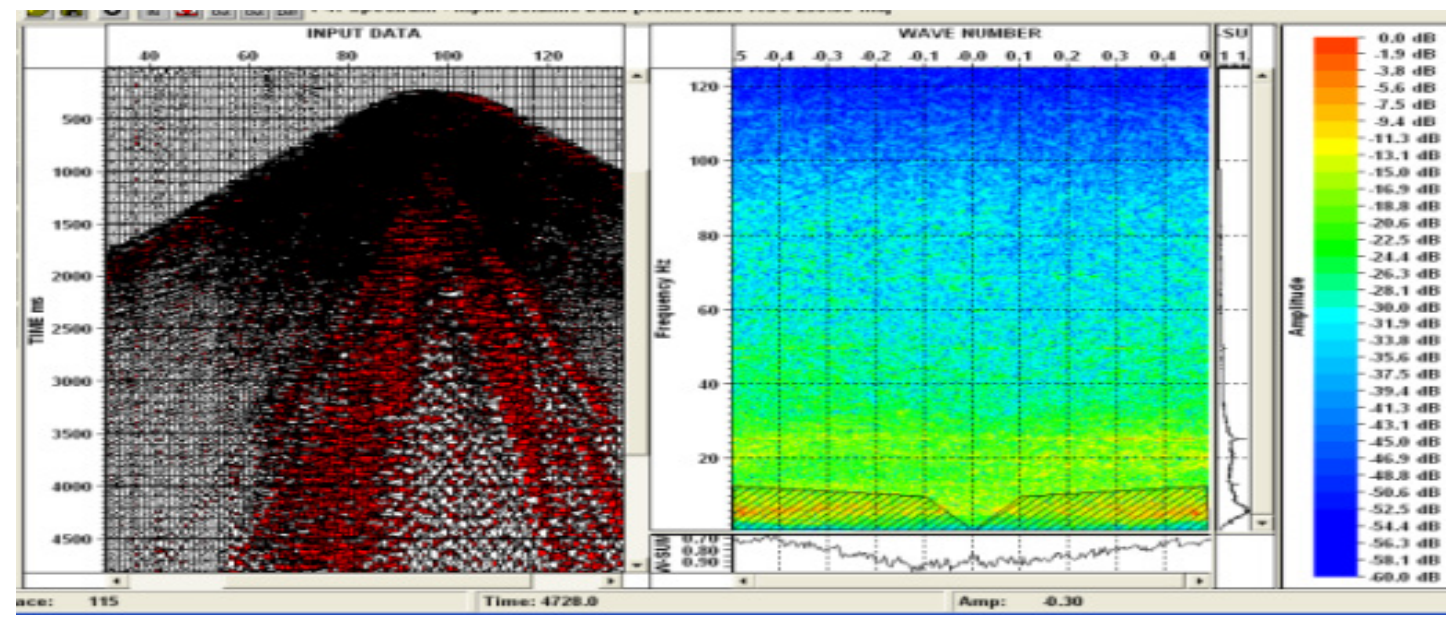

(b)

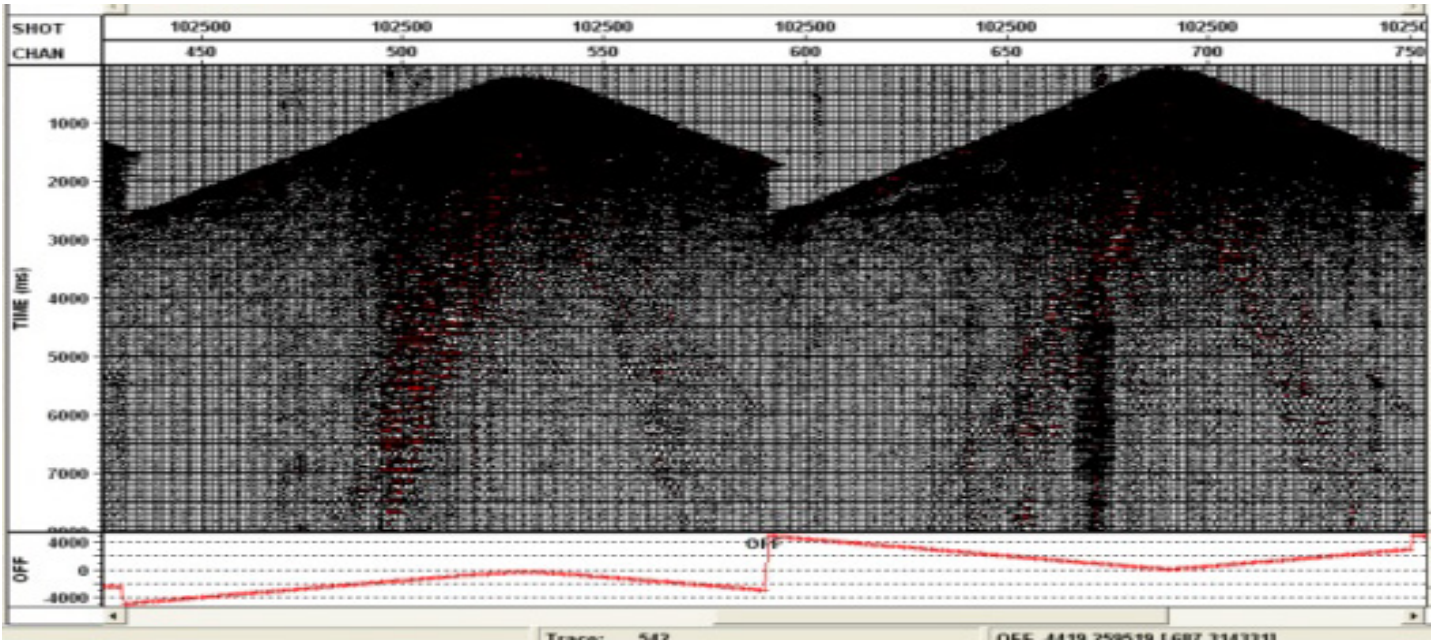

(c)

Figure 4. (a) Shows the input data with ground roll noise imprints. (b) Shows the $2^{\text {nd }}$ high pass filter designed in the F-K space juxtaposed with a panel of input data. (c) Shows the noise suppression result after the filtering operation. 


\section{Discussion of Results}

The two designed high pass $(F-K)$ filters were successfully applied to the input data and at the end of the filtering operation, the results obtained were as presented in Figures 3: (c) and 4.0: (c). The first $F-K$ filter was designed to mute (cut off) horizontally from the frequency - wave number spectrum frequencies below $15 \mathrm{~Hz}$. The choice of this cut off frequency in the designed $f-k$ filter was informed by the known characteristic low frequency of ground roll noise (usually $<15 \mathrm{~Hz}$ ) on seismic records. This filter probably, may have destroyed some useful low frequency primary reflections but this was however very minimal as primary reflection signals were still very visible and better enhanced after the filtering (Figure 3: (c)). The second $F-K$ filter was designed to mute the noise based on both low frequency and wave number $(\mathrm{K})$ basis (Figure 4: (b)). This design was informed by the fact that some useful primary reflected signals could probably still lie at low frequency regions of wave number range $\mathrm{K}=$ -0.1 to +0.1 . This second filter suppressed the ground roll appreciably but on close examination of the noise suppression result, visible traces of ground roll noise imprints were still noticed, suggesting that the second filter didn't achieve the optimal noise suppression result when compared with the first filter.

\section{Conclusions}

We have applied two high pass (low cut frequency) filters designed in $F-K$ space on a frequency - wave number analysis window to suppress dispersive ground roll noise imprints on an unprocessed onshore 3D seismic data acquired from a typical Niger delta field. The aim of the study was to suppress ground roll noise from the seismic data using the two high pass $F-K$ filters and to ascertain the optimal filter that achieved the best result for the ground roll suppression. From results obtained, the first filter achieved a better noise suppression effect than the second filter and hence is regarded as the optimal filter.

\section{Acknowledgements}

We are grateful to Shell Petroleum Development Company (SPDC) for providing the seismic datasets used for the study. We equally acknowledge GEDCO Company of Canada for the license granted to use the VISTA software tool for Academic/Research purposes at the University of Port Harcourt, Rivers State - Nigeria.

\section{REFERENCES}

[1] J. F. Claerbout (1983): Ground roll and radial traces, Stanford Exploration Project Report, SEP-35, p. 43- 53.

[2] A. Linville and R. Meck (1995): A procedure for optimally removing localized coherent noise. Geophysics; 60: 191-203.

[3] R. Saatilar and N. Canitez (1988): A Method of Ground-Roll Elimination, Geophysics; 53(\# 7): 894-902.

[4] Ö. Yilmaz and S. M. Doherty (1987): Seismic data Processing, SEG.

[5] Ö. Yilmaz (1991): Velocity-stack processing: Geophysical Prospecting; 37: 357-382.

[6] Zhou, B., and S. A. Greenhalgh (1994): Wave-equation extrapolation- based multiple attenuation: 2-D filtering in the f-k domain. Geophysics; 59: 1377-1391.

[7] R. N. Pertiwi and S. Winardhie (2012): The Application of Wiener Optimum Deconvolution Filter (WODF) with Frequency Analysis to Suppress Ground-roll in Seismic Data, Technical paper presented at the 22nd International Geophysical Conference and Exhibition, 26-29 February 2012 - Brisbane, Australia.

[8] R. Kappius and G. Crews (2001): Adaptive vector filters for ground roll reduction, CSEG Geophysics 2002 book of Abstracts. Pages 1-3. 